Artículo

\title{
Programa educativo «Trampolín» para adolescentes con problemas graves del comportamiento: perfil de sus participantes y efecto de la intervención
}

\author{
José Manuel Orrego ${ }^{\mathrm{a}, *}$, Mercedes Paino ${ }^{\mathrm{b}}$ y Eduardo Fonseca-Pedrero ${ }^{\mathrm{c}}$ \\ a Fundación Vinjoy, Oviedo, España \\ b Departamento de Psicología, Universidad de Oviedo, Oviedo, España \\ c Departamento de Ciencias de la Educación, Universidad de La Rioja, La Rioja, España
}

\section{INFORMACIÓN DEL ARTÍCULO}

\section{Historia del artículo:}

Recibido el 2 de marzo de 2015

Aceptado el 24 de junio de 2015

On-line el 1 de agosto de 2015

\section{Palabras clave:}

Trastornos del comportamiento

Problemas de conducta

Programas de intervención

Conductas disruptivas

Educación secundaria

\section{R E S U M E N}

En el Programa Trampolín participan adolescentes con graves alteraciones del comportamiento y que son considerados por las autoridades educativas como el colectivo con más dificultad para la intervención escolar ordinaria. El presente trabajo tiene 2 objetivos: a) examinar el perfil comportamental, sociodemográfico, familiar y educativo de los alumnos participantes en el Programa Trampolín y b) analizar la eficacia del programa evaluando diversos indicadores de tipo educativo y sociofamiliar. Se seleccionó una muestra de 43 alumnos del Principado de Asturias (España) ( $M=13,35$ años; DT=1,06; 41 varones) de Educación Secundaria Obligatoria. Los resultados indicaron que la muestra presentó unas características sociales, familiares y escolares específicas, que permiten identificar y describir un perfil característico de este colectivo. Asimismo, el Programa Trampolín logró mejoras en determinados indicadores considerados como adecuados para el desarrollo de estos menores. Además, se realizó una descripción pormenorizada de las respuestas utilizadas en los centros educativos.

(C) 2015 Instituto de Ciencias de la Educación de la Universidad de Oviedo. Publicado por Elsevier España, S.L.U. Este es un artículo Open Access bajo la licencia CC BY-NC-ND (http://creativecommons.org/licenses/by-nc-nd/4.0/).

\section{«Trampolín» educational programme for adolescents with severe behavioural problems: Participant profile and effect of the intervention}

\begin{abstract}
A B S T R A C T
The Trampolín Programme is intended for adolescents with severe behavioural disturbances, and who are considered by the school authorities as the group with more difficulties for ordinary educational intervention. The present study has 2 purposes: a) to examine the behavioural, sociodemographic, family and educational profile of participants in Trampolín programme; b) to analyse the efficacy of Trampolín programme, assessing several educational, social and family indicators. To do this, a selected sample of 43 secondary school pupils from Asturias (Spain) with a mean age of 13.35 years $(S D=1.06)$, of which 41 (95.3\%) were males. The study results revealed, on the one hand, that the sample had some social, family and individual characteristics, which help to identify and describe a distinctive multidimensional profile and, on the other hand, that the Trampolín Programme achieved several improvements in certain indicators considered suitable for the development of these children. In addition to this, a comprehensive description of the responses used in schools was carried out.
\end{abstract}

(C) 2015 Instituto de Ciencias de la Educación de la Universidad de Oviedo. Published by Elsevier España, S.L.U. This is an open access article under the CC BY-NC-ND license (http://creativecommons.org/licenses/by-nc-nd/4.0/)

\footnotetext{
* Autor para correspondencia: Facultad de Psicología, Universidad de Oviedo, Plaza Feijoo S/N, C.P: 33003 Oviedo (España) Tel.: +34 985103258. Correo electrónico: jm.orrego2010@gmail.com (J.M. Orrego).
} 


\section{Introducción}

A pesar de los momentos de inestabilidad propios de la edad, generalmente los adolescentes escolares se adaptan bien a las exigencias de su medio ambiente, con una conducta predecible, además de ajustada a unas reglas, cánones o normas preestablecidas (Mengíbar, 2010). La conducta y ritmo de aprendizaje de estos alumnos se adecua a las exigencias del sistema escolar, con un desarrollo relativamente armónico. Este proceso se considera indispensable para la formación de una correcta autoestima (GutiérrezSaldaña, Camacho-Calderón y Martínez-Martínez, 2007).

No obstante, con relativa frecuencia, se dan casos de estudiantes en edad escolar que no presentan un desarrollo adecuado. Esto determina y origina unos patrones cognitivos y conductuales diferentes, y esos menores suelen ser tildados de alumnos problemáticos o, más técnicamente, de menores con trastornos graves del comportamiento.

Con seguridad se puede afirmar que siempre ha existido preocupación por los problemas de conducta en el contexto escolar. No obstante, el verdadero interés seguramente se hace más palpable, en el caso español, como consecuencia de la obligatoriedad de la enseñanza hasta los 16 años, promulgada en la Ley Orgánica General del Sistema Educativo (LOGSE, 1990). La finalidad última y propedéutica de la Enseñanza Secundaria Obligatoria (ESO) choca con los intereses -o desintereses-de ciertos alumnos que no quieren participar de esa obligación y de ese derecho.

No se puede olvidar que estos menores sufren en primera persona las consecuencias de sus conductas, que afectan directamente a su desarrollo personal y humano además de a su bienestar emocional -eso por no hablar de las oportunidades vitales perdidas-. Pero este coste no solo les afecta a ellos mismos, sino que genera un importante perjuicio social. No se puede olvidar que los problemas más frecuentes que se encuentran los profesionales de la salud mental infantil son los problemas de conducta (Baker, 2013). Más aún, la tercera parte de los pacientes adolescentes que asisten a centros de salud por problemas psiquiátricos presentan algún tipo de trastornos de conducta (Perisse, Gerardin, Cohen, Flament y Mazet, 2006). Sus acciones, o más en concreto, las consecuencias que generan sus acciones, no son solo sufrimiento, impotencia y agotamiento (de las familias, seres cercanos y profesorado), sino también un gasto difícilmente cuantificable de recursos originados en la comunidad en forma de compensaciones para posibles damnificados, gastos judiciales, educativos, clínicos y, sobre todo, en la pérdida de la oportunidad para formar a una persona que puede aportar grandes beneficios a nuestra sociedad (Foster, Brennan, Biglan, Wang y al-Gaith, 2002).

Las experiencias llevadas a cabo en otras comunidades autónomas, como el Programa de Currículum Adaptado de la Fundación Ilundain en Navarra o las recomendaciones o aportaciones teóricas de innumerables profesionales en trastornos de la conducta han sido tenidos en cuenta a la hora de diseñar el modelo de intervención propio del Programa Trampolín. No obstante, las peculiaridades concretas del programa, así como la propia idiosincrasia de los participantes hacen a esta iniciativa única y original. Por todo lo cual, esta investigación se considera pertinente y necesaria para lograr un mayor conocimiento tanto de las características psicosociales de un grupo de alumnos representativo de estos problemas como para valorar las medidas concretas diseñadas, en forma de programa de intervención socioeducativa, para este colectivo de menores con graves alteraciones de la conducta.

Dentro de este contexto de investigación el presente trabajo tiene como objetivos: a) examinar el perfil comportamental, sociodemográfico, familiar y educativo de los alumnos participantes en el Programa Trampolín y b) analizar la eficacia del programa evaluando diversos indicadores de tipo educativo y sociofamiliar. Se hipotetiza que será posible extraer un perfil característico de los alumnos que acuden al Programa Trampolín. Asimismo, se hipotetiza que el Programa Trampolín tendrá un impacto en diferentes indicadores de tipo educativo y sociofamiliar.

\section{Método}

\section{El Programa Trampolín}

Para dar respuesta al cada vez mayor número de casos de alteraciones graves de la conducta entre los escolares asturianos que habían agotado todas las medidas educativas ordinarias, la Fundación Vinjoy situada en Oviedo (España) ofreció una respuesta educativa con el nombre de Programa Trampolín. Esta iniciativa fue concertada por la Consejería de Educación del Principado de Asturias (España) en el año 2006. Desde entonces, este programa realiza una intervención socioeducativa avanzada abierta y dinámica, que destaca por utilizar o rescatar tanto los métodos pedagógicos tradicionales como las nuevas corrientes educativas. Esta propuesta utiliza acciones encaminadas a promover espacios alternativos a la escuela tradicional en los cuales el alumno se vea menos influido por el estrés de estar sometido a un sistema rígido y muy reglamentado.

Trampolín fue diseñado para menores de entre 13 y 16 años con dictamen de necesidades educativas especiales (NEE) relacionadas con trastornos de la personalidad o conducta. El programa persigue como objetivo integrar nuevamente al menor en el sistema educativo o formativo-laboral, evitando su abandono prematuro. Ofrece además una formación complementaria, orientada a la mejora de las pautas de comportamiento desde el desarrollo personal y emocional. Para lograrlo los responsables del programa combinan la estancia del alumno entre Trampolín y su instituto, hasta su total integración en su centro de origen o aula ordinaria. Además, coordinan con los centros de referencia planes dinámicos y personalizados que contemplan tanto los contenidos a impartir en el programa como los tiempos que el alumno permanecerá en él. El Programa Trampolín se explica con detalle en la investigación de Orrego (2014).

\section{Participantes}

Los participantes fueron seleccionados a partir del grupo de alumnos que habían acudido al Programa de intervención socioeducativa Trampolín entre los cursos académicos 2006-2007 y 2011-2012, llevado a cabo en la Fundación Vinjoy de Oviedo. Este grupo coincide con la población total, por lo que el universo muestral lo constituyen aquellos menores que cursaron este programa hasta el curso 2011-2012. Durante este intervalo temporal pasaron por Trampolín un total de 43 alumnos, de los que 41 fueron varones $(95,3 \%)$ y 2 mujeres (4,7\%), con una media de edad en el primer momento de la intervención de 13,35 años (DT =1,06).

Las características socioeducativas comunes responden al siguiente perfil general: jóvenes adolescentes de entre 13 y 16 años que agotaron otras vías educativo-terapéuticas sin lograr los resultados esperados, pertenecientes todos ellos al contexto asturiano (domicilio habitual), adscritos a algún centro educativo de la zona central del Principado de Asturias, cursando la ESO, con dictamen de NEE o bien en proceso de elaboración y con un historial de conductas altamente disruptivas tanto en gravedad como en frecuencia. Para ingresar en el programa, además de la colaboración de las familias, se exige que el alumno se comprometa a asistir al programa de forma voluntaria, a participar en las actividades propuestas, a cumplir unas normas de convivencia mínimas y a tomar la medicación si esta le ha sido prescrita.

\section{Instrumentos}

Los instrumentos de medida utilizados fueron cuestionarios diseñados ad hoc para la presente investigación. 


\section{Cuestionarios multidimensionales}

Se confeccionó un primer cuestionario pre-post dirigido a los menores participantes en Trampolín. En él, se recogió información relacionada con el tipo de conflictividad manifestada en su entorno social, las atribuciones causales de su comportamiento, las personas con quienes tenían conflictos y su frecuencia, las causas pendientes con la justicia y su naturaleza, la frecuencia y tipo de conflictividad manifestada en clase, además de una serie de aspectos relacionados con las actitudes que el alumno tenía en su centro de referencia como la motivación hacia los estudios, el nivel de absentismo, la forma de comportarse y la tolerancia a la frustración. De igual forma, se elaboraron otros 2 cuestionarios con un formato similar al primero, pero adaptados tanto a los padres como a los orientadores de los centros de referencia. El resultado de esa triangulación fue interpretada en forma de media aritmética, en aquellos ítems cuantitativos, o también de tipo escala, mientras que los de respuesta múltiple confluían en la suma de las respuestas de los 3 colectivos. De esta forma, los valores de los ítems equivalentes de los cuestionarios multidimensionales de alumnos, familias y orientadores no se ceñían exclusivamente a las interpretaciones subjetivas de un único colectivo, sino a la convergencia de varias opiniones.

\section{Cuestionario sociodemográfico para los profesionales de Trampolín}

Por otro lado, se confeccionó un cuestionario sociodemográfico para el grupo de profesionales integrantes del Equipo Trampolín. Este recogía información relacionada con los datos de clasificación del menor y su familia, como por ejemplo la edad, sexo, lugar de nacimiento, urbanicidad, grupo étnico, situación laboral, situación económica, situación legal, estilos educativos de los padres, historia familiar, edad en el momento de la aparición de los primeros síntomas, edad cuando se produjo la primera intervención del equipo de alteraciones del comportamiento (EAC), tratamientos farmacológicos, consumo de drogas, historial escolar así como determinados aspectos posteriores a la aplicación de la medida de Trampolín como situación escolar o laboral, penal, familiar o comportamental. Para llegar a un acuerdo sobre determinadas valoraciones, se utilizó como método la discusión entre expertos. Para ello, basándose en la técnica Delphi, se utilizaron cuestionarios sucesivos con el objeto de disminuir la dispersión de las respuestas y precisar la opinión media consensuada de los profesionales del Equipo Trampolín.

\section{Procedimiento}

El Programa Trampolín fue coordinado e implementado por un grupo interdisciplinar compuesto por 5 profesionales, además de un educador-conductor, que distribuyeron o alternaron (en función de su capacitación) funciones docentes, psicológicas y pedagógicas. Respecto a la obtención de datos para la investigación, la fase previa o inicial de recogida de información fue aplicada por los anteriores profesionales del Programa Trampolín en el momento de la acogida del alumno. Durante esta primera fase los menores estuvieron acompañados por sus padres y orientadores con el fin de conocer las instalaciones donde se ubica el programa. Aprovechando esta oportunidad, se administró un instrumento de medida adaptado a los 3 colectivos, que fue cumplimentado de forma individual en las propias instalaciones del Programa Trampolín (aula o despacho acondicionado para ese fin), mediante autoinforme para alumnos y heteroinforme para padres/tutores y orientadores. Los ítems de los cuestionarios se referían al curso anterior a la incorporación del menor al Programa Trampolín. Hay que señalar que cuando no se contó con la presencia física de algún padre u orientador durante la fase de acogida, se optó por la realización de entrevistas telefónicas, que incluían los puntos del cuestionario multidimensional inicial, procurando siempre realizarlo el mismo día o los días próximos a la fecha inicial.

La evaluación post-test se llevó a cabo por los mismos profesionales del Programa Trampolín, con otros tantos cuestionarios dirigidos a los anteriores implicados, pero esta vez después de la salida del alumno del programa y coincidiendo aproximadamente con el segundo trimestre del curso siguiente. Por tanto, los ítems hacían alusión a los datos correspondientes a la mitad del curso posterior a la intervención.

La administración de los instrumentos de medida finales se realizó con los alumnos de forma presencial en las instalaciones antes descritas del programa, o en algunos casos, de forma telefónica. Respecto a los padres y orientadores se prefirió recabar los datos bien a través del teléfono, correo ordinario, email o fax.

Es conveniente señalar que no se produjo mortandad experimental pretest-postest en los colectivos alumnos y padres. No obstante, sí que hubo alguna diferencia en el grupo orientadores: algunos de ellos, al estar destinados de forma transitoria en los centros, fueron reemplazados por otros que desconocían los datos del alumno. En estos casos se obviaron los datos correspondientes a estas situaciones excepcionales, que afectaron de forma poco apreciable a los resultados finales.

\section{Análisis de datos}

En primer lugar se calcularon los estadísticos descriptivos de tendencia central y de dispersión, así como las frecuencias y porcentajes de las diferentes variables sociodemográficas, educativas y familiares analizadas en el Grupo Trampolín.

En segundo lugar, con la finalidad de examinar el tipo de medidas y respuestas punitivas y no punitivas llevadas a cabo en los centros escolares se calcularon las frecuencias y los porcentajes para cada una de ellas en el Grupo Trampolín.

En tercer lugar, para el análisis de indicadores educativos, se analizaron las frecuencias y porcentajes para 11 tipos de conflictividad en los alumnos del Grupo Trampolín de los que se habían registrado 2 medidas temporales $(n=34)$. A continuación, se examinó si existían diferencias estadísticamente significativas entre las 2 mediciones, antes y después de la intervención, tanto para las 11 medidas de conflictividad como para la variable número total de conflictos. En caso de variables dicotómicas con medición en 2 momentos temporales, se utilizó la prueba de McNemar y para la variable número total de conflictos se utilizó la prueba no paramétrica de signos de Wilcoxon. También se compararon con la prueba de Wilcoxon las puntuaciones medias autoinformadas de motivación escolar, asistencia a las clases, comportamiento correcto en clase y tolerancia a la frustración entre los 2 momentos temporales en los alumnos del Grupo Trampolín.

En cuarto lugar, se analizaron las frecuencias y porcentajes de los indicadores sociales y familiares de los que se habían registrado 2 medidas $(n=40)$. Los indicadores que se registraron fueron: diana de los conflictos, tipo de conflictos y asuntos legales. Excepto la variable frecuencia de los conflictos, dentro de agrupación diana de los conflictos, todas las variables fueron de naturaleza categórica, construyéndose a modo de resumen una puntuación total para cada una de las anteriores.

\section{Resultados}

Perfil comportamental, demográfico, familiar y educativo de los participantes

La media de edad en la que comienzan a percibirse los problemas de conducta es a los 6,95 años (DT=3,36). La primera intervención 


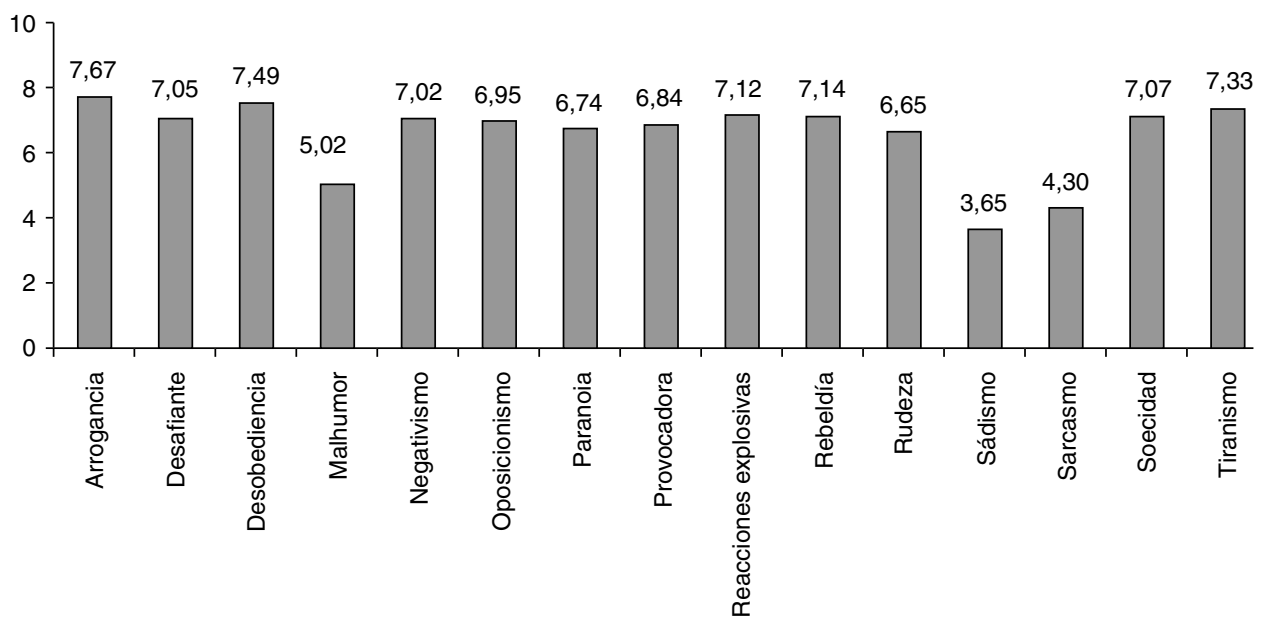

Figura 1. Puntuaciones medias en rasgos de la personalidad manifestados por los alumnos del Grupo Trampolín en función de la valoración de los profesionales.

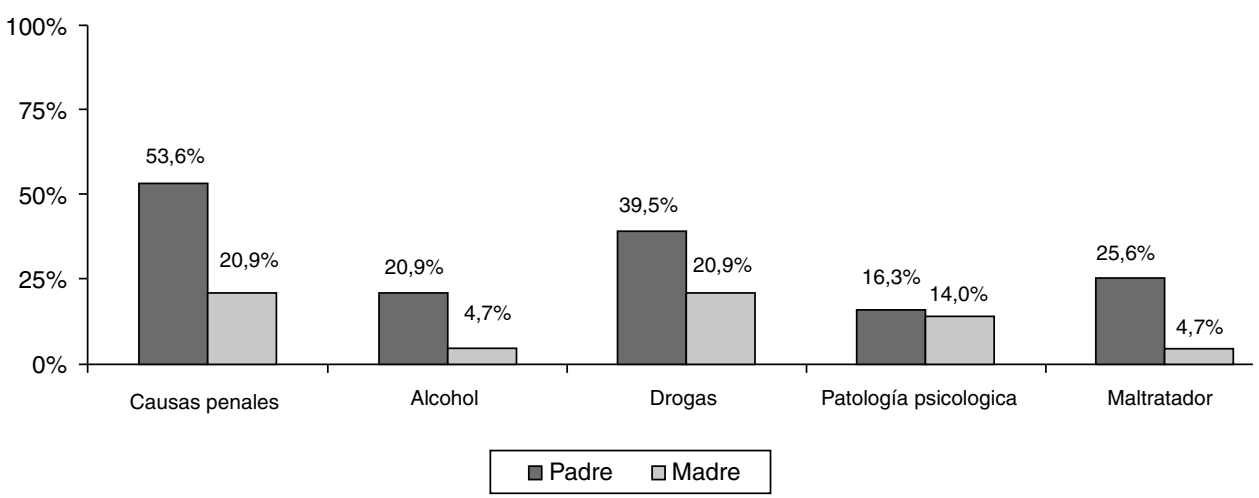

Figura 2. Porcentajes de la presencia de problemas en los progenitores de los alumnos del Grupo Trampolín.

del EAC se produce cuando los participantes tienen una media de edad de 13,35 años (DT=1,06). La diferencia de edad entre los primeros síntomas conductuales y la administración de las primeras medidas socioeducativas por parte del equipo específico de la Consejería de Educación es de 6,40 años y el número de participantes varones se corresponde con el 95,3 frente al 4,7\% de mujeres.

Respecto a las conductas disruptivas que los alumnos exhibieron durante su permanencia en el Programa Trampolín, se detallan en la figura 1 (con una escala del 1 al 10), revelando los rasgos de la personalidad que fueron más usuales. También es relevante comentar que el 90,7\% consumía algún tipo de droga y el porcentaje de ellos que acudía (o debía acudir) a salud mental durante la intervención en el programa fue del $86 \%$.

De igual forma, destacan una serie de circunstancias que se consideran singulares, como el nivel de urbanicidad. Un 74,4\% viven en las 3 principales ciudades asturianas (todas ellas superiores a los 100.000 habitantes), un $23,3 \%$ lo hacen en zonas semiurbanas (poblaciones entre 2.000 y 10.000 habitantes) y un 2,3\% tienen su domicilio en poblaciones rurales (menos de 2.000 habitantes).

Otros rasgos de interés son los relativos a su pertenencia a grupos étnicos: los datos indicaron que el $25,6 \%$ de la muestra era de etnia gitana. Respecto al nivel sociocultural de las familias es bajo 0 muy bajo en un $67,4 \%$ de los casos. Por otro lado, los valores sobre el nivel adquisitivo de las familias guardan muchas similitudes con las características socioculturales vistas anteriormente. En la figura 2 se recogen los porcentajes de los diferentes tipos de problemas o conflictividad sociofamiliar presentes en alguno de los padres de los menores (más frecuentes en el género masculino).

El ambiente familiar de la muestra estuvo determinado por una serie de características organizativas y funcionales de las que destacan los siguientes datos: la media del número de hermanos fue 1,9 (DT = 1,14); los menores del grupo de estudio Trampolín suelen ocupar en su mayoría la posición primogénita (74,4\%), mientras que el 14\% de los alumnos ocupan la segunda posición dentro del orden filial y el $7 \%$ la tercera. Los detalles de la tipología familiar se ilustran en la figura 3 y hacen mención a los diferentes modelos de convivencia en los que estaban inmersos los alumnos.

En cuanto a las diferentes tipologías educativas parentales los resultados porcentuales fueron los siguientes: las unidades familiares que manifestaron un estilo democrático fueron un $7 \%$, un estilo autoritario un $9,3 \%$ y un estilo permisivo el $39,5 \%$. No obstante hay que señalar que un $11,6 \%$ de los menores residían en el momento de la intervención en centros de acogida, por lo que estos menores quedaron fuera de la presente valoración. Además, un 32,6\% de los menores tuvieron, durante la participación en el programa, una

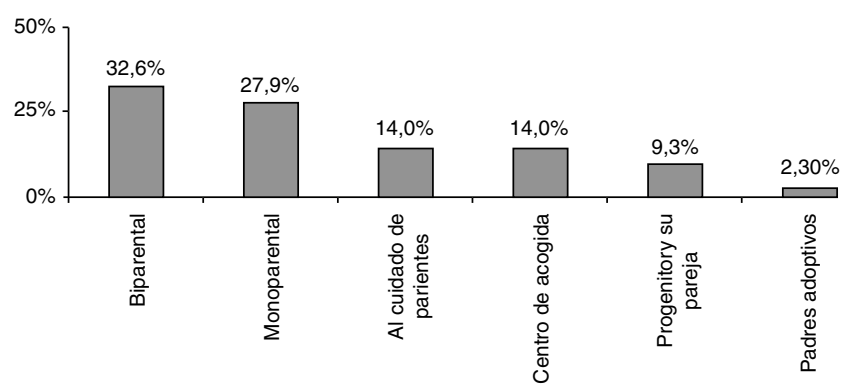

Figura 3. Porcentajes de menores del Grupo Trampolín en las estructuras familiares evaluadas. 


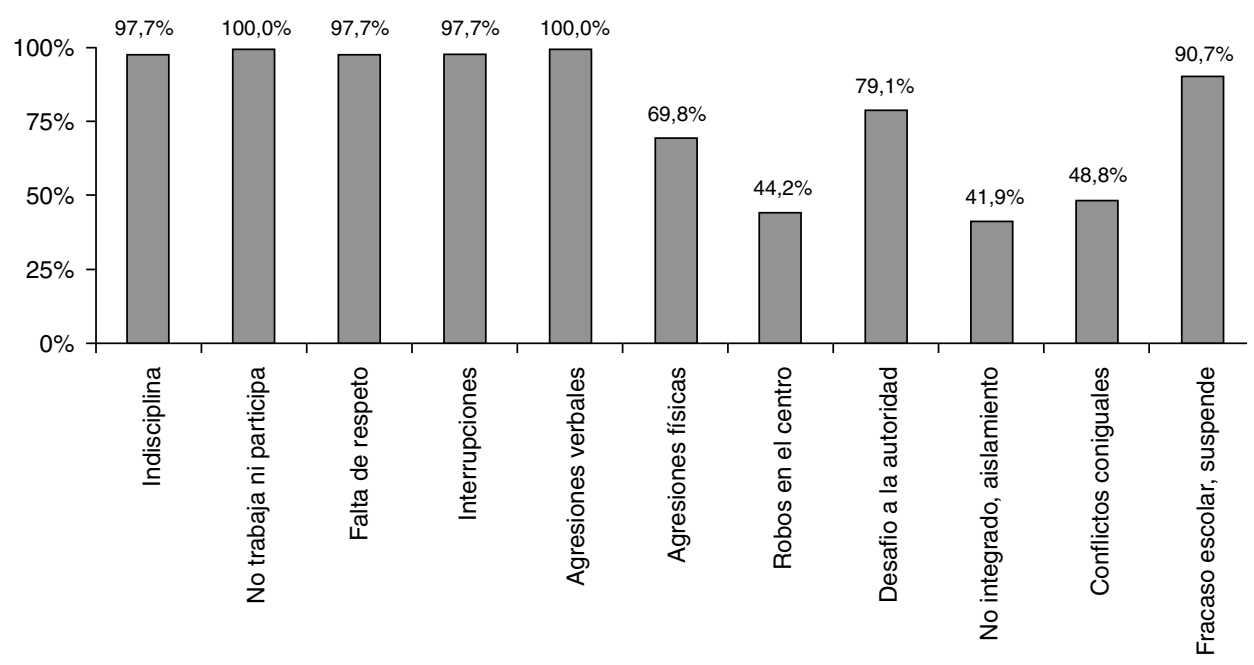

Figura 4. Porcentajes de los tipos de conductas manifestadas por el Grupo Trampolín, en el centro educativo de referencia.

estructura familiar totalmente desestructurada (carencia de figuras paternas y constantes cambios de tutela).

Una circunstancia que también ha quedado reflejada en la investigación es el grado de colaboración e implicación familiar en el seguimiento o interés por el menor. En una baremación del 1 al 5 se han constatado los siguientes resultados: el 18,6\% de las familias no se han preocupado por la evolución de su hijo; el 18,6\% han tenido una valoración baja (siendo su preocupación esporádica o poco constante); un $14 \%$ ha demostrado un interés normal, un $25,6 \%$ bueno y un $20,9 \%$ ha manifestado gran interés, participando de forma activa en el desarrollo del proceso educativo tanto en Trampolín como en el centro educativo de referencia donde el alumno cursa oficialmente sus estudios.

En cuanto a los rasgos relativos al ámbito escolar, los menores suelen cursar en institutos de enseñanza secundaria públicos en un $88,4 \%$, mientras que el $4,7 \%$ lo hacen en colegios concertados del mismo nivel educativo. Los centros, sean de un tipo u otro, suelen localizarse en entornos urbanos (79,1\%) mientras que el $20,9 \%$ corresponden a zonas semiurbanas.

En cuanto al historial educativo, el alumno de Trampolín ha repetido al menos una vez algún curso $(79,1 \%)$ y este suele ser el 1. curso de ESO. En el momento de la incorporación al programa, los menores suelen presentar adaptación curricular individualizada en varias o en todas las áreas y además estas suelen ser significativas, es decir afectan notablemente a los contenidos educativos y criterios de evaluación del alumno. Las categorías por las que los menores son dados de alta como de NEE son: por capacidad límite $2,3 \%$, trastornos de la personalidad $79,1 \%$ y déficit auditivo $2,3 \%$.

En cuanto al nivel educativo, la amplia mayoría de los participantes tiene un desfase curricular más o menos importante, que se corresponde con los siguientes ciclos educativos: un 4,7\% a primer ciclo de Primaria, un 25,6\% a segundo ciclo de Primaria, un 53,5\% a tercer ciclo de Primaria y un 11,6\% al primer ciclo de Secundaria. En cuanto a la tasas de absentismo, el EAC estimó que un 74,4\% de los alumnos que llegaron a Trampolín se podían considerar absentistas en un grado $\mathrm{u}$ otro.

Las conductas manifestadas en los centros de referencia fueron, como característica general, altamente disruptivas; además su frecuencia fue muy alta (varias veces a la semana) en un $53,5 \%$ de los casos, o bien de forma continua (todos los días) en un 46,5\% de las ocasiones. En la figura 4 se precisa con detalle la naturaleza de las actuaciones contrarias a las normas de convivencia en los centros educativos de referencia.

También se reconocen ciertas conductas comunes en los menores concernientes a diferentes formas de dominio sobre el grupo de compañeros de aula; estas características fluctúan entre actitudes de sometimiento y el abuso de poder (ya sea por la fuerza, por la amenaza o por la coacción). Los menores que, en algún momento del curso anterior a la incorporación en Trampolín, manifestaron algún episodio de violencia física en su centro educativo representaron el $69,8 \%$, frente a un $30,2 \%$ que, aunque generó situaciones conflictivas, no sobrepasó nunca ese límite. Del porcentaje de alumnos que utilizaron la agresión como mecanismo para hacer prevalecer su criterio, un $16,3 \%$ lo hizo de forma esporádica, un 25,6\% frecuentemente y un $27,9 \%$ de forma continua o sistemática.

Los centros educativos desplegaron una suerte de medidas encaminadas a la corrección del problema de comportamiento del alumno, que se resumen en la tabla 1.

\section{Valoración de la intervención del Programa Trampolín}

Después de la valoración del programa, los alumnos presentan cambios en una serie de variables relativas a indicadores socioeducativos. Sobre este aspecto destaca una reducción en la conflictividad escolar manifestada durante la permanencia en el aula para los momentos temporales antes y después de la intervención en Trampolín. Su distribución en frecuencias y porcentajes se detalla en la tabla 2 . Como se puede observar en la tabla 2 las frecuencias y porcentajes de indicadores de conflictividad se redujeron después de la intervención Trampolín, con diferencias estadísticamente significativas $(\mathrm{p} \leq 0,01)$.

Tabla 1

Respuestas educativas de los centros de referencia en los alumnos del Grupo Trampolín

\begin{tabular}{ll}
\hline Medidas punitivas & $\mathrm{z} / \mathrm{n}(\%)$ \\
\hline Amonestación verbal & $43(100)$ \\
Partes & $43(100)$ \\
Expulsiones & $43(100)$ \\
Tiempo fuera & $43(100)$ \\
No excursiones & $42(97,7)$ \\
Otras medidas & $37(86)$ \\
& \\
Medidas no punitivas & \\
\hline Horarios flexibles & $33(76,7)$ \\
Grupos flexibles & $38(88,4)$ \\
Reducción de jornada escolar & $15(34,9)$ \\
Protocolo de atención individual & $42(97,7)$ \\
Restructuración familiar & $41(95,3)$ \\
Pautas a profesores & $43(100)$ \\
Otras medidas & $6(14)$ \\
\hline
\end{tabular}


Tabla 2

Frecuencias y porcentajes de participantes de Trampolín antes y después de la intervención socioeducativa en indicadores de conflictividad dentro del aula $(n=34)$

\begin{tabular}{lll}
\hline Conflictividad escolar & $\begin{array}{l}\text { Antes } \\
\mathrm{f} / \mathrm{n}(\%)\end{array}$ & $\begin{array}{l}\text { Después } \\
\mathrm{f} / \mathrm{n}(\%)\end{array}$ \\
\hline No participa & $27(79,4)$ & $10(29,4)$ \\
Se levanta en clase & $25(73,5)$ & $7(20,6)$ \\
Interrumpe la explicación & $26(76,5)$ & $9(26,5)$ \\
Molesta a los compañeros & $26(76,5)$ & $2(5,9)$ \\
Abandona el aula sin permiso & $21(61,8)$ & $3(8,8)$ \\
Falta al respeto a los compañeros & $22(64,7)$ & $3(8,8)$ \\
Falta al respeto al profesor & $28(82,4)$ & $7(20,6)$ \\
Destroza material & $20(58,8)$ & $1(2,9)$ \\
Agrede a compañeros & $18(52,9)$ & $1(2,9)$ \\
Agrede al profesor & $22(64,7)$ & $0(0)$ \\
Otros & $8(23,5)$ & $0(0)$ \\
\hline
\end{tabular}

Tabla 3

Frecuencias y porcentajes de participantes de Trampolín antes y después de la intervención socioeducativa en indicadores de tipo de conflictividad en su centro educativo

\begin{tabular}{lll}
\hline Tipo de conflictividad & $\begin{array}{l}\text { Antes } \\
\text { f/n }(\%)\end{array}$ & $\begin{array}{l}\text { Después } \\
\mathbf{f} / \mathbf{n}(\%)\end{array}$ \\
\hline Insultos & $34(85)$ & $7(17,5)$ \\
Peleas & $29(72,5)$ & $8(20)$ \\
Pintadas & $12(30)$ & $1(2,5)$ \\
Vandalismo & $24(60)$ & $4(10)$ \\
Robos & $22(55)$ & $7(17,5)$ \\
Amenazas & $27(67,5)$ & $5(12,5)$ \\
Otros & $11(27,5)$ & $4(10)$ \\
\hline
\end{tabular}

De igual forma, también es interesante subrayar una mejora de los alumnos al final del programa respecto a las variables siguientes: motivación hacia los estudios, asistencia, corrección del comportamiento en clase y tolerancia a la frustración. Otros indicadores pre-post que fueron valorados como positivos se refieren a la variables relativas al tipo de conflictividad en su centro educativo (fuera del grupo aula). En la tabla 3 se presenta la distribución en frecuencias y porcentajes de las variables estadísticamente significativas ( $\mathrm{p} \leq 0,05)$ para los 2 momentos temporales (antes $\mathrm{y}$ después de la intervención psicoeducativa).

Sobre las atribuciones causales, se puede observar que las frecuencias y porcentajes de estas variaron después de la intervención Trampolín. Efectivamente como muestra la tabla 4, la comparación entre los 2 momentos temporales arrojó diferencias estadísticamente significativas en todos los indicadores de atribuciones $(\mathrm{p} \leq$ 0,01 ), excepto en la categoría Otros. Todos los indicadores se redujeron a excepción del referente a Trastorno psicológico, que aumentó de un 12,5 a un $75 \%$

Los resultados revelan que los adolescentes dejan de focalizar la culpa de sus problemas en factores como el fracaso escolar, el entorno social, el grupo de iguales y el funcionamiento familiar. Hay que destacar que, de todas las variables, la que cuantitativamente más se redujo fue la relativa al Grupo de iguales, luego los amigos o compañeros dejaron de ser los responsables de la mala conducta del menor. Los alumnos, por tanto, cuando finalizan en el programa,

\section{Tabla 4}

Frecuencias y porcentajes de participantes de Trampolín antes y después de la intervención socioeducativa en indicadores de atribuciones causales

\begin{tabular}{lll}
\hline Atribuciones causales & $\begin{array}{l}\text { Antes } \\
\mathrm{f} / \mathrm{n}(\%)\end{array}$ & $\begin{array}{l}\text { Después } \\
\mathrm{f} / \mathrm{n}(\%)\end{array}$ \\
\hline Trastorno psicológico & $5(12,5)$ & $30(75)$ \\
Fracaso escolar & $18(45)$ & $6(15)$ \\
Entorno social & $20(50)$ & $6(15)$ \\
Grupo de iguales & $31(77,5)$ & $8(20)$ \\
Funcionamiento familiar & $15(37,5)$ & $4(10)$ \\
Otros & $2(5)$ & $2(5)$ \\
\hline
\end{tabular}

atribuyen mayoritariamente la causa de su conflictividad a razones psicopatológicas o clínicas.

\section{Discusión}

Las particularidades del grupo de alumnos Trampolín permitieron el conocimiento de un colectivo de alumnos identificado como el más complejo en cuanto a problemas graves de comportamiento, en el contexto de la educación secundaria del Principado de Asturias. La identificación de ciertas características relativas a las dimensiones personal, familiar, social y educativa resulta de gran utilidad para todos los profesionales que trabajan tanto con menores en situación de riesgo social o fracaso escolar como en el ámbito terapéutico y educativo en general.

Respecto a la dimensión personal, una peculiaridad distintiva del Grupo Trampolín es la corta edad en la que surgen los problemas graves de conducta. Este dato revela una relativa precocidad y, por lo tanto, la posibilidad de efectuar un tratamiento tanto profiláctico como preventivo. Este aspecto es compartido por diversos autores (González-Menéndez, Fernández-Hermida y Secades,2004; SabriàPau, 2004; Van-Goozen y Fairchild, 2006) que inciden en la importancia de la anticipación de las intervenciones para asegurar su efectividad. En la muestra analizada en Trampolín, más del 95\% de los menores fueron varones. Estas cifras confirman la mayor prevalencia del sexo masculino con problemas graves del comportamiento de tipo externalizante. Además los estudios de expertos como Chun y Mobley (2010), Emberley y Pelegrina (2011), Eme (2007), Fonseca-Pedrero, Lemos-Giráldez, Paino, y Muñiz (2011), Hartung, Lefler y Fedele (2011), López-Soler, Castro, Alcántara, Fernández y López (2009). Trepat y Ezpeleta (2011) ponen de manifiesto las diferencias comportamentales entre géneros, sobre todo en lo referente a conductas desadaptativas o comportamientos agresivos. En este sentido, la influencia del sexo, aunque no pueda llegar a considerarse como determinante, sí puede ser considerada como un factor de riesgo a la hora de padecer una alteración grave del comportamiento. Los profesionales de Trampolín pudieron percibir y valorar una serie de conductas relacionadas con los trastornos graves del comportamiento que resultaron frecuentes en los alumnos. Dichas conductas fueron: arrogancia, conductas desafiantes, desobediencia, malhumor, negativismo, oposicionismo, paranoia, conductas provocadoras, reacciones explosivas, rebeldía, rudeza, sadismo, sarcasmo, uso de expresiones soeces y conductas tiránicas. Existen investigaciones que relacionan los trastornos graves de la conducta con la expresión de este tipo de manifestaciones (Andreu, Peña y Penado, 2013; Ballesteros-Alcalde, 2004; Emberley y Pelegrina, 2011; Javaloyes y Redondo-Romero, 2005; Labrador, 2008; Pardini y Fite, 2010; Rojas-Marcos, 2005), por lo que esta coincidencia viene a apoyar el perfil de los menores que participan en el Programa Trampolín.

En cuanto a la dimensión familiar, los modelos o núcleos familiares son biparentales o monoparentales en aproximadamente el $50 \%$ de los casos. Están en situación de convivencia con parientes cercanos, centros de acogida o situaciones cercanas a la exclusión social el resto de los alumnos. Se puede afirmar que prácticamente la mitad de los integrantes del Grupo Trampolín tienen o han tenido una situación de desestructuración familiar. Diversos estudios (Castellano-Barca, 2002; Raya, Pino, Ruiz y Herruzo, 2013; Tur, Mestre-Escrivá y del-Barrio-Gándara, 2004) ponen el acento en la relación entre las prácticas parentales deficientes, los ambientes familiares adversos y los trastornos graves del comportamiento. Se confirma, por tanto, que los ambientes familiares desestructurados presentes en numerosos casos en el grupo de estudio Trampolín, pronostican o aumentan las probabilidades de que los menores padezcan un trastorno del comportamiento grave. En cuanto al nivel sociocultural de las familias del programa, los datos 
mostraron que más del $80 \%$ de las familias tenían un nivel entre bajo y muy bajo. En la misma línea, el nivel económico de las familias fue también bajo o muy bajo. Estudios como los de Ary, Duncan, Duncan, y Hops (1999), Rutter y Guiller (1988) o Tur et al. (2004) vinculan diversas características sociofamiliares, entre ellas la clase social, con las conductas antisociales de los hijos, por lo que la pertenencia a un nivel social bajo constituye un predictor de riesgo relacionado estrechamente con los problemas de conducta. También se ha detectado alta frecuencia de conflictos graves intrafamiliares que se concretan en episodios de maltrato por parte del cabeza de familia, tropiezos con la justicia, consumo de sustancias o psicopatologías de alguno de los progenitores o miembros del núcleo consanguíneo. Autores como Satterfield y Schell (1997) han valorado la asociación entre los ambientes de riesgo y los problemas de conducta en la infancia con la probabilidad de convertirse en un delincuente en la edad adulta. Si tenemos en cuenta que las conductas en general, y en particular aquellas de riesgo, surgen como imitación de otras copiadas del entorno más próximo, nos encontramos que el núcleo familiar puede ser un indicador premonitorio. No obstante, es necesario recordar que las familias disfuncionales no garantizan que los hijos desarrollen conductas anómalas, sino que estas situaciones se desencadenan cuando convergen ciertas particularidades en el menor relacionadas con problemas psicológicos latentes.

Respecto a los aspectos sociales de los alumnos del programa, uno de los más evidentes es la urbanicidad: las $3 / 4$ partes de los alumnos tienen su domicilio en zonas urbanas. Investigaciones como las llevadas a cabo por LeClair (2001) relacionan las áreas altamente urbanizadas con mayores tasas de problemas de comportamiento. Las causas pueden hallarse en que las poblaciones con menor densidad de población favorecen el seguimiento y control de los menores por parte de la comunidad y, con ello, la disminución de los conflictos. No obstante, una objeción a la anterior hipótesis puede ser que la población se concentra en la zona central de la región y, además, está dentro del área de alcance del transporte del Programa Trampolín: quizá estos sean en realidad los factores determinantes para interpretar el anterior aspecto social. Otra cuestión importante es la referente al consumo de sustancias que nos indica que más del 90\% de los menores del Grupo Trampolín consume algún tipo de droga de forma habitual, siendo el alcohol y el cannabis las sustancias más consumidas. Son numerosas las investigaciones que relacionan los trastornos graves del comportamiento con conductas de riesgo como el consumo de drogas (Bernal y González-Tascón, 2009; Carli et al., 2014; Gutiérrez et al., 2008; Howell, 2003; Plattner et al., 2012; Quinsey, Skilling, Lalumière y Craig, 2004); estos estudios coinciden con los resultados hallados en Trampolín sobre este aspecto concreto, por lo que se puede afirmar que los menores del programa tienen como característica común el ser consumidores habituales tanto de sustancias socialmente aceptadas (alcohol y tabaco) como de otras consideradas ilegales.

Continuando con los aspectos escolares, los participantes en Trampolín mayoritariamente pertenecen a centros públicos. Las causas sobre por qué los centros concertados no demandan ayuda a las autoridades educativas no están claras y deberían ser objeto de estudio. No obstante, y justificando esa futura línea de investigación, alguna de esas reticencias pudieran estar relacionadas con una menor matrícula de alumnos con problemas de conducta, intervenciones socioeducativas más adecuadas con este perfil de alumnado, mayor grado de tolerancia de los profesores, encubrimiento de los problemas por parte de la dirección u otras causas aún sin determinar. Desde el punto de vista académico, más de las $3 / 4$ partes de los alumnos fueron repetidores. El primer curso de ESO fue el más habitual para repetir, seguido de 6 . $^{\circ}$ de educación primaria. Además, más del 80\% de los menores tenían dictamen de NEE. Prácticamente todos ellos eran por trastornos de la personalidad. Las adaptaciones curriculares significativas se llevaron a cabo en un $72,1 \%$ de los casos. En cuanto al desfase curricular, este se correspondió a un retraso escolar de al menos 2 cursos. Otro rasgo característico de los participantes en el programa es que casi las $3 / 4$ partes de los menores fueron absentistas en un grado u otro. Las conductas que los alumnos manifestaron en sus centros de referencia, y que originaron su derivación a la experiencia Trampolín, fueron prácticamente en todos los casos agresiones verbales, ausencia de trabajo, rechazo a participar en clase, indisciplina, falta de respeto e interrupciones constantes. Las agresiones físicas también fueron usuales en un $69,8 \%$, así como las manifestaciones de desafío al profesor $(79,1 \%)$. Con estos resultados no es de extrañar que la tasa de fracaso escolar sobrepasara el $90 \%$.

Por todo lo anterior y a modo de conclusión, una vez conocidas las particularidades del grupo de estudio Trampolín, es factible delinear un perfil característico de este alumno participante en el programa. Las peculiaridades distintivas permiten concluir que, atendiendo a los datos vistos, un menor en esta edad de escolarización tiene un problema grave de conducta cuando manifiesta comportamientos no deseados por la sociedad, se opone a las normas establecidas, deteriora la convivencia social, educativa o familiar y además puede llegar a ser diagnosticado con algún trastorno de la personalidad, que afecta directamente a la esfera comportamental. Por otro lado, siguiendo los planteamientos de Lemos-Giráldez (2003), el contexto ambiental donde se desenvuelve este menor puede aumentar o disminuir la frecuencia y probabilidad de aparición de patrones disfuncionales de conducta, por lo que haber sido criado en ambientes desestructurados o haber experimentado episodios traumáticos no es condición sine qua non para desarrollar un trastorno de conducta, sino que cuando confluyen esas circunstancias con la existencia de conductas latentes los problemas pueden llegar a materializarse.

Respecto a la valoración de los resultados pre-post obtenidos a raíz de la intervención desarrollada en el Programa Trampolín se observaron mejoras significativas en una serie de indicadores de índole escolar además de otras variables autoinformadas que versaban sobre la motivación hacia los estudios, la asistencia a clase, el grado de corrección del comportamiento y la tolerancia a la frustración. En cuanto a estos 2 últimos indicadores y siguiendo investigaciones como la llevada a cabo por Samper, Tur, Mestre y Cortés (2008), que reveló que los adolescentes con altos niveles de agresividad utilizaban mayoritariamente estilos de afrontamiento improductivo, mientras que sus iguales más prosociales se centraban en estrategias basadas en la resolución de problemas, se deduce que los alumnos participantes en el programa lograron adquirir habilidades para solucionar ciertos problemas sin necesidad de utilizar recurrentemente la violencia.

Además se percibieron también mejoras en otros indicadores de tipo sociofamiliar que tuvieron que ver con el objeto diana de los conflictos o destinatarios principales de las agresiones infligidas por el menor. Antes de la intervención, los alumnos del Grupo Trampolín focalizaron principalmente los conflictos en los profesores y fueron estos el colectivo diana más perjudicado. Aunque no fue objeto de estudio sí se percibió que los alumnos dirigían su hostilidad hacia algunos profesores concretos, generalmente hacia aquellos que demandaban más exigencia, o que reclamaban unos conocimientos previos o de base para poder impartir sus materias (como por ejemplo los profesores de inglés o matemáticas). También resultaron más vilipendiados aquellos profesores implicados en alguna expulsión o aquellos que, a juicio de los menores, fueron los causantes de alguna sanción injusta. La capacitación más adecuada del manejo de las interrelaciones, el control de las emociones y reacciones y, sobre todo, de la capacidad de empatía de los alumnos de Trampolín provocó que los profesores y en general las personas cercanas al menor dejaran de ser el blanco constante de los ataques tanto físicos como verbales. 
Otro progreso observado en los menores, derivado de la intervención en Trampolín y muy llamativo por su impacto social, fue la reducción de las conductas contrarias a las normas sociales. Después de la participación en el programa los menores mejoraron significativamente en todas las variables analizadas, reduciéndose los porcentajes a menos de la mitad en todos los indicadores estudiados. También es destacable el hecho de que las conductas problemáticas más frecuentes fueron las relacionadas con las agresiones y también con la amenaza. Luego, si se parte del concepto de que estas manifestaciones de violencia no son instintivas, sino que se adquieren y se aprenden, se puede pensar que el proceso tiene la capacidad de invertirse y se puede llegar a su desactivación, como así se persiguió con la intervención.

Por todo lo anterior, el Programa Trampolín se puede considerar una opción socioeducativa adecuada para el tratamiento de este tipo de problemas con adolescentes en contextos análogos al estudiado.

En cuanto a las limitaciones del estudio, conviene aclarar que otros factores pudieron interferir en la evaluación del programa, como por ejemplo el tipo de diseño experimental o la ausencia de grupo control. El cambio actitudinal de los menores pudo verse influido por otras variables ajenas a la intervención realizada. Estas se refieren a los procesos naturales de maduración relacionados con la adolescencia, que como es sabido pueden frenar o acelerar cualquier avance psicopedagógico. De la misma forma, otras intervenciones paralelas (servicios sanitarios, fiscalía de menores u otras iniciativas privadas) pudieron ser agentes de influencia. No obstante, las evidencias en cuanto a historias personales de otros casos similares sugieren que los factores antes citados raramente influyen en el itinerario de estos alumnos. Por todo lo anterior, el Programa Trampolín se considera como causante factible del cambio positivo en el colectivo reseñado. Las características del alumnado, explicadas en el apartado Participantes, sobre el que se presume que el Programa Trampolín ha sido eficaz, son a la vez que singulares necesarias para que el programa surta el efecto deseado. Si no se dieran las condiciones exigidas (negativa a asistir, falta de colaboración familiar o no tomar la medicación) - por poner algún ejemplo-, difícilmente se podría realizar una intervención como la descrita en este texto.

\section{Conflicto de intereses}

Los autores declaran no tener ningún conflicto de intereses.

\section{Agradecimientos}

Esta investigación pudo llevarse a cabo gracias a la colaboración desinteresada de la Fundación Vinjoy y del Equipo de Alteraciones del Comportamiento de la Consejería de Educación del Principado de Asturias. También es pertinente aludir al proyecto referencia: MICINN-12-PSI2011-23818.

\section{Referencias bibliográficas}

Andreu, J. M., Peña, M. E. y Penado, M. (2013). Impulsividad cognitiva, conductual y no planificadora en adolescentes agresivos reactivos, proactivos y mixtos. Anales de psicología, 29(3), 734-740.

Ary, D. V., Duncan, T. E., Duncan, S. C. y Hops, H. (1999). Adolescent problem behavior: The influence of parents and peers. Behaviour Research and Therapy, 37(3) $217-230$.

Baker, K. (2013). Conduct disorders in children and adolescents. Paediatrics and Child Health, 23(1), 24-29.

Ballesteros-Alcalde, M. C. (2004). Trastornos del comportamiento en la adolescencia: evaluación clínica individual y familiar. Monografías de psiquiatría, 16(1), 21-33.

Bernal, J. y González-Tascón, M. (2009). Medidas de prevención situacional en la nueva cultura del ocio juvenil: especial referencia a las experiencias desarrolladas en Asturias. Revista de derecho penal y criminología, 3(1), 215-260.
Carli, V., Hoven, C. W., Asserman, C. W., Hiesa, F. C., Guffanti, G., Sarchiapone, M., et al. (2014). A newly identified group of adolescents at «invisible» risk for psychopathology and suicidal behavior: Fndings from the SEYLE study. World Psychiatry, 13(1), 78-86.

Castellano-Barca, G. (2002). Factores protectores en el desarrollo psicosocial de niños y adolescentes. A tu Salud, revista de educación para la salud, 39, 7-10.

Chun, H. y Mobley, M. (2010). Gender and grade-level comparisons in the structure of problem behaviors among adolescents. Journal of Adolescence, 33(1), 197-207.

Emberley, E. y Pelegrina, M. (2011). Prevalencia, sintomatología y distribución del trastorno negativista desafiante. Psicothema, 23(2), 215-220.

Eme, R. F. (2007). Sex differences in child-onset, life-course-persistent conduct disorder. A review of biological influences. Clinical Psychology Review, 27(5), 607-627.

Fonseca-Pedrero, E., Lemos-Giráldez, S., Paino, M., Santarén-Rosell, M., SierraBaigrie, S. y Ordóñez-Camblor, N. (2011). Instrumentos de medida para la evaluación del fenotipo psicótico. Papeles del Psicólogo, 32(2), 129-151.

Foster, S. L., Brennan, P., Biglan, A., Wang, L. y al-Gaith, S. (2002). Prevención de problemas de conducta: lo que sí funciona. Series: prácticas educativas, 8, 1-43.

González-Menéndez, A., Fernández-Hermida, J. R. y Secades, R. (2004). Guía para la detección e intervención temprana con menores en riesgo. Gijón: Colegio Oficial de Psicólogos del Principado de Asturias.

Gutiérrez-Saldaña, P., Camacho-Calderón, N. y Martínez-Martínez, M. (2007). Autoestima, funcionalidad familiar y rendimiento escolar en adolescentes. Atención Primaria, 39(11), 597-603.

Gutiérrez, M. C., Santamaría, A., Manuel-Gutiérrez, A., Bernabé, E., Arranz, P., GarcíaBenaite, D., et al. (2008). Trastorno de déficit de atención e hiperactividad: un reto compartido. Semergen Medicina de Familia, 5, 230-234.

Hartung, C.M., Lefler, E.K., Fedele, D.A. (2011). Disruptive behaviors and aggression. En: Brown, B.B. y Prinstein, M.J. (Eds.), Encyclopedia of adolescence (pp. 143149). San Diego: Academic Press.

Howell, J. C. (2003). Preventing and reducing juvenile delinquency. Thausand Oaks, CA:: Sage Publications.

Javaloyes, A. y Redondo-Romero, A. (2005). Trastorno del comportamiento: trastorno negativista desafiante, trastorno disocial y otros problemas del comportamiento. Pediatría Integral, 6, 27-37.

Labrador, F. J. (2008). Técnicas de modificación de conducta. Madrid: Pirámide.

LeClair, J. A. (2001). Children's behaviour and the urban environment: An ecological analysis. Social Science E' Medicine, 53(3), 277-292.

Lemos-Giráldez, S. (2003). La psicopatología de la infancia y la adolescencia: consideraciones básicas para su estudio. Papeles del Psicólogo, 85, 19-28.

LOGSE. (1990). Boletín Oficial del Estado núm. 238, de 4 de octubre de 1990. Madrid: Gobierno de España.

López-Soler, C., Castro, M., Alcántara, M., Fernández, V. y López, J. A. (2009). Prevalencia y características de los síntomas externalizantes en la infancia. Diferencias de género. Psicothema, 21, 353-358.

Mengíbar, C. (2010). El adolescente busca su propia identidad. Innovación y experiencias educativas, 32, 1-9.

Orrego, J. M. (2014). Perfil psicosocial y modelo de intervención en adolescentes con problemas graves del comportamiento. España: [Tesis de doctorado no publicada], Universidad de Oviedo, Facultad de Psicología.

Pardini, D. A. y Fite, P. J. (2010). Symptoms of conduct disorder, oppositional defiant disorder, attention-deficit/hyperactivity disorder, and callous-unemotional traits as unique predictors of psychosocial maladjustment in boys: Advancing an evidence base for DSM-5. Journal of the American Academy of Child \& Adolescent Psychiatry, 49(11), 1134-1144.

Perisse, D., Gerardin, P., Cohen, D., Flament, M. y Mazet, P. (2006). Le trouble des conduites chez l'enfant et l'adolescent: une revue des abords thérapeutiques. Neuropsychiatrie de l'Enfance et de l'Adolescence, 54(8), 401-410.

Plattner, B., Giger, J., Bachmann, F., Brühwiler, K., Steiner, H., Steinhausen, H., et al. (2012). Psychopathology and offense types in detained male juveniles. Psychiatry Research, 198(2), 285-290.

Quinsey, V. L., Skilling, T. A., Lalumière, M. L. y Craig, W. M.(2004).Juvenil delinquency: Understanding the origins of individual differences. Washington, DC: American Psychological Association.

Raya, A. F., Pino, M. J., Ruiz, R. y Herruzo, J. (2013). Relationship between parenting style and aggression in a Spanish children sample. Procedia, 82, 529-536.

Rojas-Marcos, L. (2005). Las semillas de la violencia. Madrid: Espasa- Calpe.

Rutter, M. y Guiller, H. (1988). Delincuencia juvenil. Barcelona: Martínez- Roca.

Sabrià-Pau, J. (2004). Detección precoz de los problemas emocionales y de conducta en el niño. Pediatría Integral, 8(8), 642-650.

Samper, P., Tur, A. M., Mestre, V. y Cortés, M. T. (2008). Agresividad y afrontamiento en la adolescencia. Una perspectiva intercultural. International Journal of Psychology and Psychological Therapy, 8(3), 431-440.

Satterfield, J. y Schell, A (1997). A prospective study of hyperactive boys with conduct problems and normal boys: Adolescent and adult criminality original research article. Journal of the American Academy of Child E'Adolescent Psychiatry, 36(12), 1726-1735

Trepat, E. y Ezpeleta, L. (2011). Sex differences in oppositional defiant disorder. Psicothema, 23(4), 666-671.

Tur, A., Mestre-Escrivá, V. y del-Barrio-Gándara, V. (2004). Los problemas de conducta exteriorizados e interiorizados en la adolescencia: relaciones con los hábitos de crianza y con el temperamento. Acción Psicológica, 3(3), 207-221.

Van-Goozen, S. y Fairchild, G. (2006). Neuroendocrine and neurotransmitter correlates in children with antisocial behavior. Hormones and Behavior, 50(5), 647-654. 